\title{
From Current Researches to Future Applications
}

\author{
Vladimir I. Chizhik ${ }^{1} \cdot$ Murat S. Tagirov $^{2}$
}

Published online: 14 May 2018

(C) Springer-Verlag GmbH Austria, part of Springer Nature 2018

Any, even at the first glance quite abstract, research ultimately provokes interests because of possible applications in the activities of humanity. The main current tendency is to turn scientific knowledge into technological products. Applications of the magnetic resonance (MR) to study condensed media were realized just after the registration of the electron spin resonance (EPR, 1944), nuclear magnetic resonance (NMR, 1946), and nuclear quadrupole resonance (NQR, 1950) in these objects. Magnetic resonance has also been successfully used for quantitative and qualitative analysis (evaluation of the quality of food, estimation of oil and gas productivity of rocks, and much more).

The goal of the special issue is to reflect a few interesting aspects of modern trends in NMR, which may bring new methodology developments and important practical applications. The first three articles are devoted to the further progress of the NMR technique.

Solid-state NMR spectroscopy has found wide applications after the development of the method of magic-angle spinning (MAS) that allowed obtaining highresolution spectra in solid and viscous samples. In the article, "Magic-angle spinning NMR and molecular mobility in heterogeneous systems" D. Michel treated the problem of applicability of the method to heterogeneous systems with nanometer dimensions. The special situation is considered when local magnetic fields at interphases are not completely averaged out even in the case of fast thermal motion of embedded molecules. At first, the local inhomogeneous magnetic fields, which arise

Vladimir I. Chizhik

v.chizhik@spbu.ru

Murat S. Tagirov

Murat.Tagirov@kpfu.ru

1 Saint Petersburg State University, Saint Petersburg, Russia

2 Kazan Federal University, Kazan, Russia 
near polycrystalline grains in porous media, are described. Then, it is shown that the application of MAS NMR for heterogeneous systems may lead to the appreciable increase in spectral resolution even in the cases when the local internal motion is characterized by relatively short correlation times. Several examples are given in which the combined influence of MAS and thermal motion becomes obvious.

The article of M. Cifelli, V Domenici, V. I. Chizhik, and S. V. Dvinskikh ${ }^{215} \mathrm{~N}-{ }^{13} \mathrm{C}$ dipole couplings in a smectic mesophase of a thermotropic ionic liquid" is devoted to a new approach to access ${ }^{15} \mathrm{~N}-{ }^{13} \mathrm{C}$ dipolar couplings with much higher sensitivity and greater spectral resolution. Structural information in rigid and soft solids can be obtained by NMR spectroscopy through the measurements of dipole-dipole spin couplings, which are very sensitive to interatomic distances. The dipolar interaction is also orientationally dependent and, thus, reports on molecular dynamics at the atomic level. Experimental detection of the ${ }^{15} \mathrm{~N}-{ }^{13} \mathrm{C}$ dipolar splitting at the natural abundance level is challenging due to the combination of the two rare isotopes: the fraction of ${ }^{15} \mathrm{~N}-{ }^{13} \mathrm{C}$ coupled pairs is, in fact, only $4 \times 10^{-5}$. Nevertheless, in a straightforward but long $(48 \mathrm{~h})$ experiment with the 4-pentyl-4'cyanobiphenyl (5CB) nematic phase, the ${ }^{13} \mathrm{C}$-satellites were earlier observed, for example, in the NAN15 spectrum. The authors demonstrate that well-resolved spectra can be recorded within $10 \mathrm{~h}$ of experimental time using a standard commercial NMR probe and a moderately strong magnetic field. The technique is applied to a thermotropic smectic mesophase formed by an ionic liquid with imidazolium-based organic cation.

A new approach for the interpretation of high-resolution NMR spectra is proposed in the article of S. I. Selivanov "Comparison of relative differences of experimental and calculated vicinal constants ${ }^{3} J_{\mathrm{H}-\mathrm{H}}$ for conformational analysis of rigid molecules in liquids". Successful application of NMR spectroscopy in structural and conformational analysis of molecules in liquid phase is based on measuring and correct interpretation of spin-spin coupling constants (SSCC, $J$ ) between magnetic nuclei in molecules under investigation. This approach requires knowledge of the dependences of $J$ values on any parameters of molecular structure. Among them the relationship between vicinal scalar constant ${ }^{3} J$ and dihedral angle $\theta$ is most well known and widely used in practice. For the first time, dependence ${ }^{3} J=f(\theta)$ was defined and described for the case of proton-proton scalar interactions $\left({ }^{3} J_{\mathrm{HH}}\right)$ in substituted ethanes by Martin Karplus. During a few decades original, so-called, "Karplus relationship" has been widely discussed and modified by scientists many times. The main result of theoretical discussions and experimental studies is the introduction into the initial Karplus equation of additional terms describing electronegativity and relative orientation of substituents. A lot of special modifications of Karplus relationship were suggested and used for conformational analysis of local groups of molecules or for investigation of their separate fragments. As empirical parameters in all local modifications are determined by fitting corresponding equation to the experimental data derived from a single-type molecular system, the applicability of such a Karplus equation to other systems has been limited in general. The main sources of differences between experimental and calculated values of ${ }^{3} J_{\mathrm{HH}}$ are usually poor parameterization and specific time-averaging of experimental data at fast (in NMR time-scale) exchange between some conformers. In Selivanov's article, it 
is suggested to use the comparison of experimental and calculated relative differences of vicinal SSCCs instead of usual comparison of absolute values of experimental and calculated vicinal constants. This approach theoretically may eliminate different inputs into $\Delta^{3} J_{\mathrm{HH}}$ errors and increase sensitivity of ${ }^{3} J_{\mathrm{HH}}$ data to conformational states of molecules in a liquid.

Being the most universal method for structural investigation in physics, chemistry, and biology, NMR has also been extended to medical applications that are reflected in two articles.

One of the most prominent applications of NMR is magnetic resonance imaging (MRI). Today, MRI added another dimension to NMR and is now well established as the most informative medical diagnostic method. MRI is the ideal method for preclinical imaging, non-invasive biomedical and pharmaceutical research on animals. Pathological processes can be followed over a long time period on one single animal. This gives much more reliable results and saves laboratory animals and costs. However, there are still diseases which are purely understood and where all imaging methods including MRI have almost no diagnostic value. The connected problems are discussed in the article of U. Eichhoff "Can MRI contribute to the understanding and therapy of mental diseases?" The author tries to evaluate the possible contribution of Diffusion Tensor Imaging (DTI) and resting state functional MRI to a better understanding of these diseases. The nowadays most accepted hypothesis of the origin of mental diseases is an impaired connectivity between various brain areas. DTI and MR tractography reveal structural connectivities through neuronal fibers between brain areas. Functional Imaging in the Resting State (rs-fMRI) allows ones to visualize functional connectivities. In this method, the time course of rapidly repeated MRI scans is evaluated through the Blood Oxygen-Level Dependent (BOLD) contrast. Statistical evaluation and cross correlation of the signals in all voxels show synchrony of signal level fluctuations even in remote brain areas. Since mental diseases often represent highly individual features associated with the patient personality, these initial findings by rs-fMRI should be regarded as a possible key to a better understanding of the nature and origin of mental diseases.

The Cuban experience in quantitative magnetic resonance associated with molecular, preclinical and clinical studies of significant diseases and drug developments is presented in the article of C. Cabal Mirabal, A. Fernández García, M. Lores Guevara, E. González, and L. Oramas Díaz "Kinetics studies of complex biomedical process by magnetic resonance. Cuban experiences". As examples, "in vitro" and "in vivo" MR studies of Sickle Cell disease, the Diabetic Foot Ulcer, the Brain Tumor Response and the Magnetic Nano Particles Pharmacokinetics, are presented. Furthermore, contributions and restrictions of magnetic resonance to diagnostic and optimization of therapeutic pathways are discussed in some particular cases.

It is very rare in science that a new material, discovered theoretically by physicists, would be almost immediately confirmed experimentally, and, moreover, would quickly find a lot of extremely promising applications in technologies or any practical applications. A very young, but already, very "hot" direction of research in physics is topological isolators (TIs). TIs have unusual properties: these materials, in general, have low electric conductivity in bulk like semiconductors or insulators but there is an important exception: these materials have the high surface conductivity 
of the metallic type. Such properties of TIs make these materials as new candidates for using in spintronics and magnetoelectronic devices and also for building up quantum computers. The article of A. O. Antonenko, D. Yu. Nefedov, E. V. Charnaya, S. V. Naumov, and V. V. Marchenkov is devoted to the investigation of semiconductor $\mathrm{Bi}_{2} \mathrm{Se}_{3}$ which is a $3 \mathrm{D}$ topological insulator: " ${ }^{77} \mathrm{Se}$ low temperature NMR in the $\mathrm{Bi}_{2} \mathrm{Se}_{3}$ single crystalline topological insulator". The authors observed two component NMR spectra at the three temperatures (15.8, 88, and $293 \mathrm{~K})$. It has been shown that the NMR spectrum almost does not shift with decreasing temperature and the density of charge carriers did not follow the thermal activation law.

Two articles are connected with problems of ecology and "green chemistry". Ionic liquids (ILs), as a relatively new class of liquid system, have been investigated actively during the last few decades. NMR is one of the well-known techniques for studying the mobility in various liquid systems, including imidazolium-based ILs. Amino acid ionic liquids (AAILs) are a novel class of chiral ionic liquids, which properties expand significantly the field of their application in various technologies. Compared with the conventional ionic liquids, AAILs are considered as more biodegradable and biocompatible, less toxic and can enhance stability of biomaterials. AAILs were tried to utilize as a reaction medium in gas separation or heterogeneous catalysis or as a selector in the chiral liquid extraction etc. An example of the investigation of transport properties of two long-chain AAILs is presented in the article of Yu. S. Chernyshev, A. S. Koneva, E. A. Safonova "NMR self-diffusion study of amino acid ionic liquids based on 1-methyl-3-octylimidazolium in water".

Self-assembly and structural transitions in amphiphilic systems play an important role in various processes: from production of nanostructured materials to transport in biological cells. Their properties are the result of unique molecular structures of amphiphilic substances, i.e. each molecule contains both a hydrophilic and a hydrophobic part. In aqueous solutions above a certain concentration (critical micelle concentration, CMC), surfactants form a variety of organized assemblies: micelles, vesicles, and lyotropic liquid crystals. Therefore, they have been the subject of numerous experimental and theoretical studies. Gemini surfactants are very popular ones, their interfacial activity and molecular aggregation properties can widely be modified by adjusting the three structural elements that characterize them (two polar head groups, two aliphatic chains, and one spacer). Gemini surfactants broadly apply in various fields, like soil remediation, separation of biomaterials, enhanced oil recovery, and drug entrapment and release. Aggregation of the decyltrimethylammonium bromide (DTAB) and cetyltrimethylammonium bromide (CTAB) in $\mathrm{D}_{2} \mathrm{O}$ has been studied by M. V. Popova and D. L. Raev. The results are reflected in the article "Aggregation behavior of monomeric surfactants and a gemini cationic surfactant by NMR and computer simulation data".

We hope that the publications presented in this issue will attract the attention of scientific community to the prospects of new MR applications and will stimulate further progress in the magnetic resonance and its applications. We would like to bring our gratitude to all authors who have contributed to this special issue. 